\title{
Nanometer-Scale Characterization Tools for Strain-Engineered Semiconductor Devices
}

\author{
V. B. Ozdol ${ }^{1}$, C. T. Koch $^{2}$ and A. M. Minor ${ }^{1,3}$ \\ 1. National Center for Electron Microscopy, Lawrence Berkeley National Laboratory, CA, USA \\ 2. Institut für Experimentelle Physik, Universität Ulm, Germany \\ 3. Department of Materials Science and Engineering, University of California, Berkeley, CA, USA
}

The performance of today's state of the art semiconductor electronic devices depends on charge transport within very small volumes of the active device regions. One means of optimizing the band structure of the device as well as enhancing the charge carrier mobility is to tune its strain state. Being able to map strain with high spatial resolution and a large field of view is therefore crucial for developing modern microelectronic devices. Electron microscopy is still the only technique to offer the required spatial resolution. In this contribution, we will focus on image-based techniques such as highresolution transmission electron microscopy (HRTEM) [1] and dark-field inline holography (DIH) [2] as they are able to provide higher spatial resolution compared to diffraction-based methods.

HRTEM-based strain mapping relies on the assumption that every unit cell across the field of view has the same contrast, allowing distortions in the observed pattern to be interpreted as lattice distortions. Figure 1a shows an HRTEM image of a 22-nm FinFET device with uniform contrast across the field of view. Figure 1b displays color-coded two-dimensional strain maps calculated using GPA [1]. For this particular structure, there is no pronounced deformation observed in both longitudinal $(0.0 \pm 0.2) \%$ and vertical direction $(-0.3 \pm 0.2) \%$. However, there is significant bending $(2.3 \pm 0.2)^{\circ}$ of the right fin with respect to the reference crystal.

While HRTEM is quite efficient for mapping relatively short-ranged strain fields induced by features limited in size, it becomes much more difficult if the strain fields extend beyond the field of view of a single HRTEM image (typically $100 \times 100 \mathrm{~nm}^{2}$ ). To overcome this limitation, DIH technique [2,3] has been developed. As an alternative to dark-field off-axis holography (DOAH) [4], DIH relies on the reconstruction of the geometric phase from a focal series of zero-loss filtered dark-field images using an exit-wave reconstruction algorithm. The major advantages of DIH over DOAH are the lack of need for a defect-free reference area as well as very loose requirements on the spatial coherence of the illumination and potentially better signal/noise properties for the same electron dose. Figure 2 a shows the experimental setup and contrast mechanism in DIH. Figure $2 \mathrm{~b}$ displays sub-areas of three differently focused and zero-loss filtered (0002) dark-field images of the LED structure consisting of six InGaN QWs. The geometric phase and the displacement field are reconstructed using the FRWR algorithm. Figure $2 \mathrm{c}$ shows the out-of-plane strain map across the whole active area of the LED revealing the well width fluctuations.

In this contribution we will cover the guidelines for quantitative strain analysis including the TEM sample preparation requirements [5], the optimization of HRTEM imaging and the reliable reconstruction of the geometric phase from focal series of dark field images.

References:

[1] M. J. Hÿtch, E. Snoeck, and R. Kilaas, Ultramicroscopy 74, 131 (1998)

[2] C.T. Koch, V.B. Özdöl, P.A. van Aken, Appl. Phys. Lett. 96, 091901 (2010) 
[3] V.B. Özdöl, C.T. Koch, P.A. van Aken, J. Appl. Phys 108, 056103 (2010)

[4] M. J. Hÿtch, et al., Nature 453, 1086 (2008)

[5] V. B. Özdöl, V. Srot, and P. A. van Aken, in Handbook of Nanoscopy, Wiley-VCH (2012)
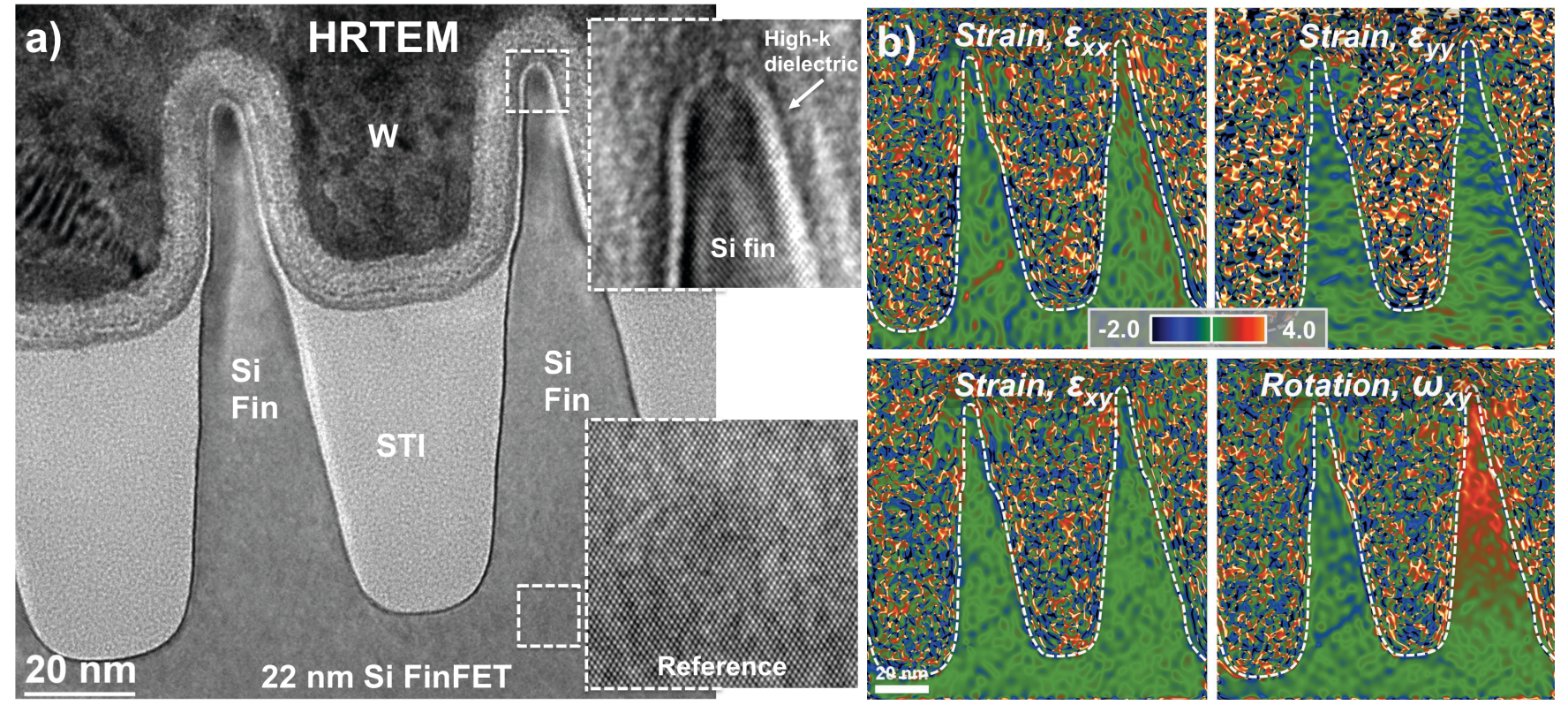

Figure 1a HRTEM image of a 22-nm FinFET transistor. b Two-dimensional strain maps of FinFET structure calculated using GPA: Longitudinal, $\varepsilon_{\mathrm{xx}}$, vertical $\varepsilon_{\mathrm{yy}}$, and shear strain, $\varepsilon_{\mathrm{xy}}$, as well as crystal rotation, $\omega_{\mathrm{xy}}$.

a)
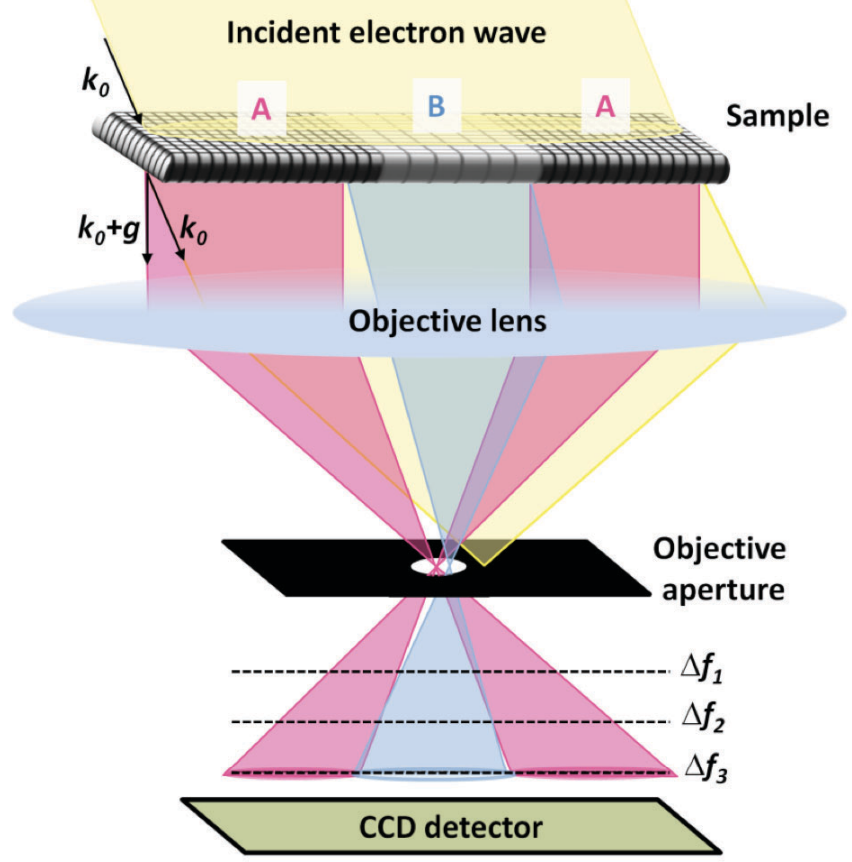
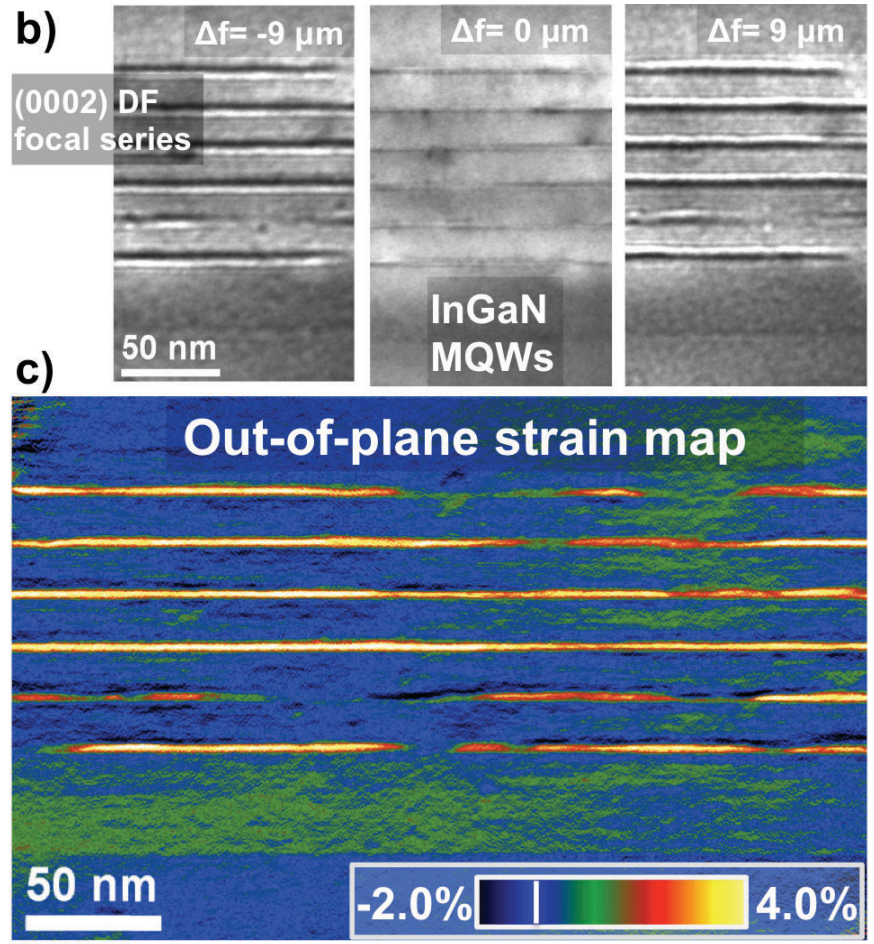

Figure 2a Diagram illustrating the principle of DIH. Quantitative strain analysis on InGaN-based LED devices: b (0002) dark-field focal series (3 images out of 11 are shown here) recorded using an automated data acquisition procedure. c Out-of-plane strain map of the active area of the LED structure. 